\title{
Comparison of GENIE and Conventional Supervised Classifiers for Multispectral Image Feature Extraction
}

\author{
Neal R. Harvey, James Theiler, Steven P. Brumby, Simon Perkins, John J. Szymanski, Jeffrey J. Bloch, Reid B. Porter, \\ Mark Galassi, and A. Cody Young
}

\begin{abstract}
We have developed an automated feature detection/classification system, called GENetic Imagery Exploitation (GENIE), which has been designed to generate image processing pipelines for a variety of feature detection/classification tasks. GENIE is a hybrid evolutionary algorithm that addresses the general problem of finding features of interest in multispectral remotely-sensed images. We describe our system in detail together with experiments involving comparisons of GENIE with several conventional supervised classification techniques, for a number of classification tasks using multispectral remotely sensed imagery.
\end{abstract}

Index Terms-Evolutionary algorithms, genetic programming, image processing, multispectral imagery, remote sensing, supervised classification.

\section{INTRODUCTION}

$\mathbf{L}$ ARGE volumes of remotely sensed multispectral data are being generated from an increasing number of increasingly sophisticated airborne and spaceborne sensor systems. While there is no substitute for a trained analyst, exploitation of this data on a large scale requires the automated extraction of specific features of interest. Creation and development of task-specific feature-detection algorithms is important, yet can be extremely expensive, often requiring a significant investment of time and effort by highly skilled personnel.

Our particular interest is the pixel-by-pixel classification of multispectral remotely-sensed images, not only to locate and identify but also to delineate particular features of interest. These range from broad-area features such as forest and open water to man-made features such as buildings and roads. The large number of features in which we are interested, together with the variety of instruments with which we work, make the hand-coding of suitable feature-detection algorithms impractical. We are therefore using a supervised learning approach that can, using only a few hand-classified training images, generate image processing pipelines that are capable of distinguishing features of interest from the background. We remark that our approach is to consider the two-class problem: although many applications require the segmentation of an image into a larger number of distinct land-cover types, we consider the simpler problem of identifying a single class against a background of "other" classes.

Manuscript received January 23, 2001; revised June 29, 2001. This work was supported by the U.S. Departments of Energy and Defense.

The authors are with the Los Alamos National Laboratory, Los Alamos, NM 87545 USA (e-mail: harve@lanl.gov).

Publisher Item Identifier S 0196-2892(02)01565-6.
In applying general-purpose supervised learning techniques to multispectral imagery, the usual approach is to employ purely spectral input vectors, formed by the set of intensity values in each spectral channel for each pixel in the image. These vectors provide a convenient fixed-dimensionality space in which conventional classifiers can often work well. It is clear, however, that spatial relationships (such as texture, proximity, or shape, all of which are disregarded with purely spectral vectors) can be very informative in scene classification. Many different kinds of extra spatial context information could be added to the spectral information, as additional dimensions of the pixel input vector. The problem is that there exists a combinatorically vast choice for these additional vector dimensions; yet it is clear that a suitable choice of additional dimensions could make classification much easier. Unfortunately, this suitable choice is, in general, application-specific.

To address this problem, we have developed a hybrid evolutionary algorithm called GENetic Imagery Exploitation (GENIE) [2]-[8], that searches through the space of image processing algorithms. GENIE is a hybrid in that the evolutionary part of the program attempts to identify a pipeline of image processing operations which transform the raw multi-spectral data planes into a new set of image planes; these intermediate "scratch" planes are then used as input to a conventional supervised classification technique to provide the final classification results.

When adopting an evolutionary approach, a critical issue is the representation of candidate solutions in order that they may be effectively manipulated. We use a genetic programming (GP) method of representation of solutions, due to the fact that each individual will represent a possible image processing algorithm. GP has previously been applied to image-processing problems, including: edge detection [9], film restoration [10], face recognition [11] and image segmentation [12]. The work of Daida et al. [13] and Bandyopadhyay and Pal [14] (as well as our own work, cited above) is of particular relevance since it demonstrates that GP can be employed to successfully evolve algorithms for real tasks in remote-sensing applications.

The beauty of an evolutionary approach is its flexibility: all that is required is a representation for candidate solutions, a fitness measure for comparing candidate solutions, and a scheme for "mutating" candidate solutions into other candidate solutions. Many varied problems beyond image processing have been successfully solved using evolutionary computation, from optimizing of dynamic routing in telecommunications networks [15] to designing protein sequences with desired structures [16], and many others. 
This paper describes our system in detail together with experiments involving comparisons of GENIE with several conventional supervised classification techniques, for a number of classification tasks using multispectral remotely-sensed imagery.

The remainder of the paper is organized as follows: Section II describes the GENIE system in detail. Section III describes the conventional supervised classification techniques with which GENIE is to be compared. Section IV describes the data and classification tasks on which the algorithms are to be tested and compared. Section V describes the results of the comparisons. Section VI describes further comparison with multiclass versions of the supervised classifiers. Finally, Section VII discusses these results and concludes.

\section{THE GENIE SySTEM}

GENIE employs a classic evolutionary paradigm: a population is maintained of candidate solutions (chromosomes), each composed of interchangeable parts (genes), and each assessed and assigned a scalar fitness value, based on how well it performs the desired task. After fitness determination, the evolutionary operators of selection, crossover and mutation are applied to the population and the entire process of fitness evaluation, selection, crossover and mutation is iterated until some stopping condition is satisfied.

\section{A. Training Data}

The environment for each individual in the population consists of data planes, each of these planes corresponding to a separate spectral channel in the original image, together with a weight plane and a truth plane. The weight plane identifies those pixels to be used in training - these are the pixels for which the analyst is confident in identifying as either "true" and "false": true defines areas where the feature of interest exists; false defines areas where that feature does not exist. The actual delineation of true and false pixels is given by the truth plane. This arrangement permits us the flexibility (not used in this study) to employ both real-valued weights (representing degrees of confidence or of importance) and real-valued truth (corresponding to retrieval of continuous valued properties). The data in the weight and truth planes may be derived from actual ground truth (collected on the ground, at or near the time the image was taken) or from the best judgement of an analyst looking at the data. Because collecting ground truth data is so expensive, our system employs a graphical interface called ALADDIN to assist the analyst in making judgements about and marking out features in the data. The analyst can view a multispectral image in a variety of ways, and can create training data by painting directly on the image using a computer mouse. Fig. 1 shows an image alongside the markup that an analyst provides as "ground truth." Figs. 4(b), and 6(b) show further examples where the analyst has marked out the desired feature on the image.

\section{B. Encoding Candidate Solutions}

Each individual chromosome in the population consists of a fixed-length string of genes. Each gene in GENIE corresponds to a primitive image processing operation. Therefore the entire

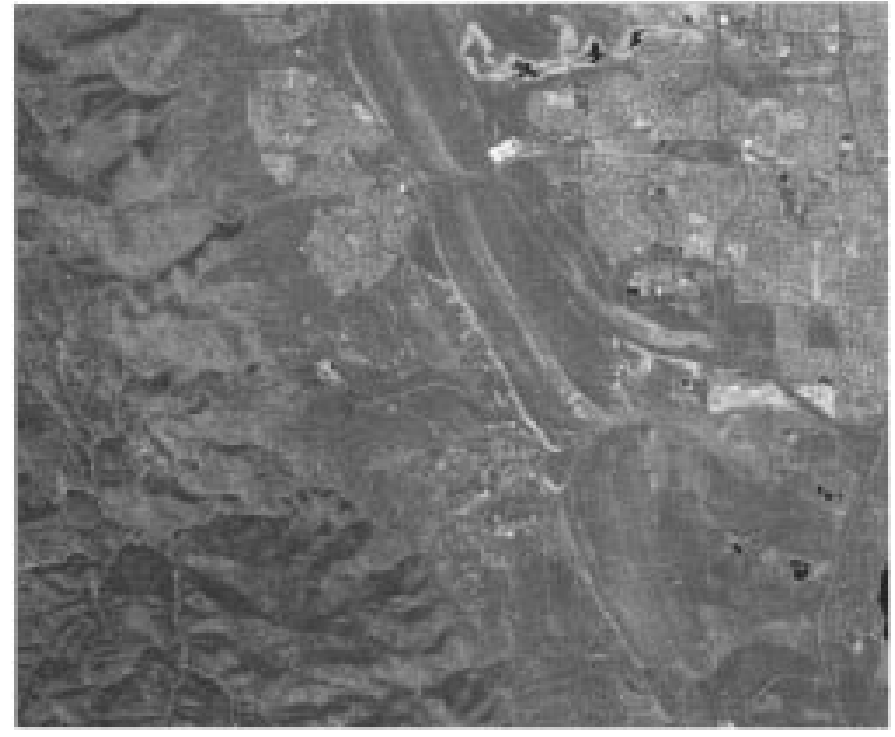

(a)

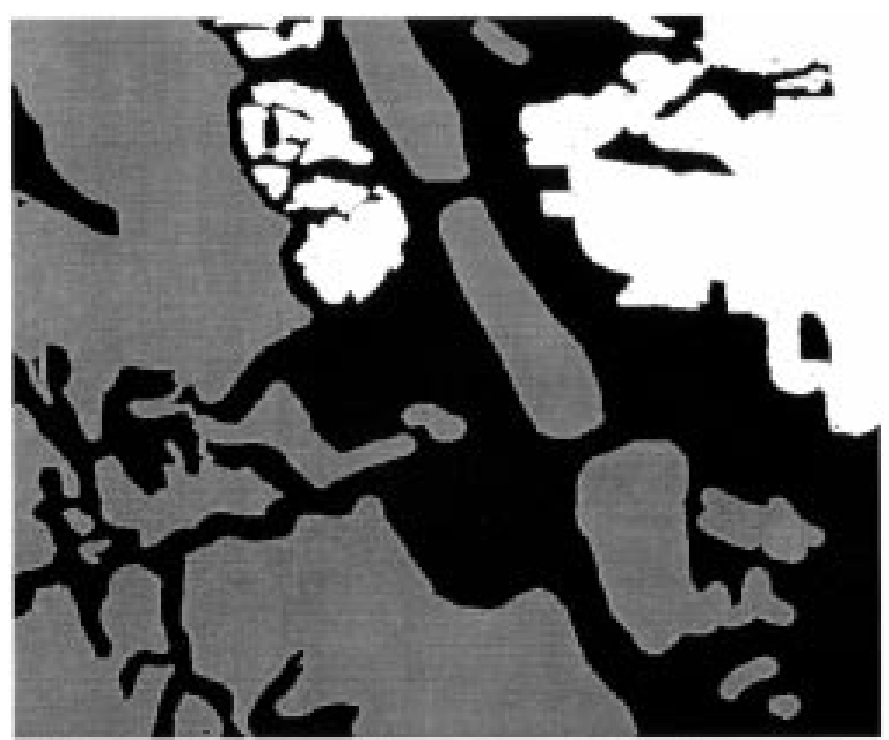

(b)

Fig. 1. (a) Greyscale images of one of the scenes used to produce the training data for "Urban Areas" (Urban 1). (b) Training data provided for the training scene for "Urban Areas" (white = feature, grey = not feature, and black = no assertion).

chromosome describes an algorithm consisting of a sequence of primitive image processing operations.

A single gene consists of an operator name, a list of input planes, specifying from which plane input is to come; a list of (usually one) output plane; and a list of scalar parameters. Parameters may be integer, floating point, or categorical. Each gene used in GENIE takes one or more distinct image planes as input, and produces one or more image planes as output. Input can be taken from any data planes in the training data image cube. Output is written to any of a small number of scratch planes-temporary workspaces where an image plane can be stored. Genes can also take input from scratch planes, but only if that scratch plane has been written to by another gene earlier in the chromosome sequence. 
TABLE I

Primitive Image Processing Operators (GENES) Used IN GENIE AND What They Do

\begin{tabular}{|c|c|c|c|}
\hline $\begin{array}{c}\text { Gene } \\
\text { Abbreviation } \\
\end{array}$ & $\begin{array}{c}\text { Image } \\
\text { Processing } \\
\text { Operation } \\
\end{array}$ & $\begin{array}{c}\text { Inputs/ } \\
\text { Outputs/ } \\
\text { Parameters } \\
\end{array}$ & Notes \\
\hline $\begin{array}{l}\text { ADDP } \\
\text { ADDS } \\
\text { SUBP } \\
\text { DIFF } \\
\text { NDI } \\
\text { MULTS } \\
\text { NEG } \\
\text { MULTP } \\
\text { SQRT } \\
\text { SQR } \\
\text { LINSCL } \\
\text { LINCOMB } \\
\end{array}$ & $\begin{array}{c}\text { Add planes } \\
\text { Add scalar } \\
\text { Subtract planes } \\
\text { Absolute difference } \\
\text { Normalized difference } \\
\text { Multiply by scalar } \\
\text { Negate plane } \\
\text { Multiply planes } \\
\text { Square root } \\
\text { Square } \\
\text { Linear scale } \\
\text { Linear combination } \\
\end{array}$ & $\begin{array}{l}2 / 1 / 0 \\
1 / 1 / 1 \\
2 / 1 / 0 \\
2 / 1 / 0 \\
2 / 1 / 0 \\
1 / 1 / 1 \\
1 / 1 / 0 \\
2 / 1 / 0 \\
1 / 1 / 0 \\
1 / 1 / 0 \\
1 / 1 / 2 \\
2 / 1 / 1 \\
\end{array}$ & $\begin{array}{l}\text { Basic mathematical operations. ADDS adds a scalar, which may } \\
\text { be negative, to its input. DIFF is like SUBP but outputs the } \\
\text { absolute values. NDI is like SUBP, but divides the result by the } \\
\text { sum of its two inputs. MULTS scales its input by a scalar, which } \\
\text { by default is positive. LINSCL is like MULTS but takes an extra } \\
\text { parameter which is added onto the scaled input. LINCOMB } \\
\text { outputs a linear combination of its two inputs, in proportion } \\
\text { specified by its one parameter, which takes a value between } 0 \\
\text { and } 1 .\end{array}$ \\
\hline $\begin{array}{l}\text { MIN } \\
\text { MAX } \\
\text { IFLTE }\end{array}$ & $\begin{array}{c}\text { Minimum } \\
\text { Maximum } \\
\text { 'If less than else' }\end{array}$ & $\begin{array}{l}2 / 1 / 0 \\
2 / 1 / 0 \\
4 / 1 / 0\end{array}$ & $\begin{array}{l}\text { Logical operations. MIN and MAX perform pixel-wise minimum } \\
\text { and maximum, equivalent to AND and OR for binary input. } \\
\text { IFLTE outputs its third input wherever the first input is less } \\
\text { than its second input, and its fourth input elsewhere. }\end{array}$ \\
\hline $\begin{array}{l}\text { SAVAR } \\
\text { SADIF } \\
\text { SADIST } \\
\text { SANORM }\end{array}$ & $\begin{array}{l}\text { Spectral angle variance } \\
\text { Spectral angle difference } \\
\text { Spectral angle distance } \\
\text { Normalize spectral vector }\end{array}$ & $\begin{array}{c}2-16 / 1 / 2 \\
2-16 / 1 / 2 \\
2-10 / 1 / 2-10 \\
2-10 / 2-10 / 0\end{array}$ & $\begin{array}{l}\text { Spectral angle operations. SAVAR and SADIST look at two } \\
\text { circular neighborhood regions around each pixel, of size defined } \\
\text { by their two parameters. SAVAR returns the difference between } \\
\text { the variance of the spectral angles of the pixels in the two } \\
\text { regions. SADIF returns the difference between the mean spectral } \\
\text { angle of both regions. SADIST returns the spectral angle } \\
\text { difference between each pixel and the vector defined by its } \\
\text { parameters. SANORM normalizes the vector defined by its } \\
\text { inputs to have a magnitude equal to dataScale. }\end{array}$ \\
\hline QTREG & Region Size related to Statistics & $1 / 3 / 1$ & $\begin{array}{l}\text { QTREG Determines the region size (in log base } 2 \text { ) around each } \\
\text { pixel for which the normalized variance per pixel standard of the } \\
\text { square region first reaches a given threshold. Also returns planes } \\
\text { with the linear fit slope and offset of the variance as a function } \\
\text { of region scale for each pixel }\end{array}$ \\
\hline
\end{tabular}

The image processing algorithm represented by any particular chromosome can be thought of as a directed acyclic graph, where the nonterminal nodes are primitive image processing operations, and the terminal nodes are individual image planes extracted from the multispectral image used as input. The scratch planes are the "glue" that combines primitive operations into image processing pipelines. Traditional GP [17] uses a variable sized (within limits) tree representation for algorithms. Our representation differs in that it allows for reuse of values computed by subtrees, since many nodes can access the same scratch plane, i.e., the resulting algorithm is a graph rather than a tree. It also differs in that the total number of nodes is fixed.
Our notation for genes is most easily illustrated by an example: the gene [ADDP rD1 rS1 wS2] applies pixel-by-pixel addition to two input planes, read from data plane 1 and from scratch plane 1, and writes its output to scratch plane 2. Additional operator parameters, if any, are listed after the input and output arguments.

Our "gene pool" is composed of a set of primitive image processing operators which we consider useful. For different applications, the user may want to choose different sets of primitive operators; for the studies described here, we used the operators described in Table I. These include spectral, spatial, spatio-spectral, logical and thresholding operators. 
The set of morphological operators is restricted to function-set processing morphological operators, i.e., gray-scale morphological operators having a flat structuring element. The shape of the structuring elements used by these operators is chosen from among: square, circle, diamond, horizontal cross and diagonal cross, and horizontal, diagonal, and vertical lines. The shape and size of the structuring element are defined by operator parameters. Other local neighborhood/windowing operators such as mean, median, etc. specify their kernels/windows in a similar way. The spectral operators have been chosen to permit weighted sums, differences, and ratios of data and/or scratch planes.

It should be noted that although all chromosomes have the same fixed number of genes, the effective length of the resulting algorithm graph may be smaller than this. For example, an operator may write to a scratch plane that is then overwritten by another gene before anything has a chance to read from it. GENIE performs an analysis of chromosome graphs when they are created and only carries out those processing steps that actually affect the final result. Therefore, the fixed length of the chromosome acts as a maximum effective length.

In an interesting parallel to "junk DNA" in natural chromosomes, the final chromosomes produced by GENIE often exhibit some redundancy, i.e., genes and answer planes that do not contribute to the answer. While these "junk genes" do not affect the functionality of the chromosome, they can make it harder to understand how the chromosome works. We have therefore developed a simple postrun pruning process that removes junk genes and ineffective answer planes from the final solution if this is required.

\section{Backends}

Final classification requires that the algorithm produce a single scalar output plane, which can then be thresholded to produce a binary output. It would be possible to treat, for example, the contents of scratch plane $\mathbf{S 1}$ as the output from the algorithm (thresholding of this plane may be required to obtain a binary result). However, we have found it advantageous to adopt a hybrid approach which applies a conventional supervised classifier to a (sub)set of scratch and data planes to produce the final output plane.

To do this, we first select a subset of the scratch and data planes to be answer planes. The conventional supervised classifier "backend" uses the answer planes as input and produces a final output plane; in principle, we can use any supervised classification technique as the backend but for the comparisons reported here, we used the Fisher Linear Discriminant [20]. This provides a linear combination of the answer planes that maximizes the mean separation between true and false pixels, normalized by the total variance in the projection defined by the linear combination. The output of the discriminant-finding phase is a continuous-valued (gray-scale) image, which is then reduced to a binary image by finding the threshold value that maximizes the fitness as described in the following section.

\section{Fitness Evaluation}

The fitness of a candidate solution is given by the degree of agreement between the final binary output plane and the training data. If we denote the detection rate (fraction of "true" pixels

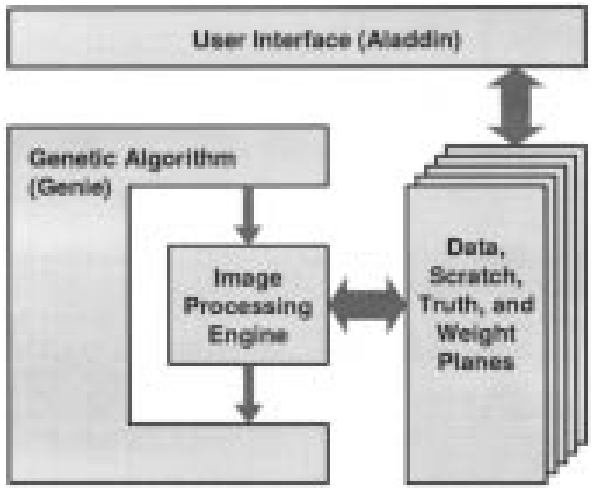

Fig. 2. Software architecture of the GENIE System.

classified correctly) as $R_{d}$ and the false alarm rate (fraction of "false" pixels classified incorrectly) as $R_{f}$, then the fitness F of a candidate solution is given by

$$
F=500\left(R_{d}+\left(1-R_{f}\right)\right) .
$$

Thus, a fitness of 1000 indicates a perfect classification result. This fitness score gives equal weighting to type I (true pixel incorrectly labeled as false) and type II (false pixel incorrectly labeled as true) errors. Note a fitness score of 500 can be trivially achieved with a classifier that identifies all pixels as true (or all pixels as false).

\section{E. Software Implementation}

The evolutionary algorithm code has been implemented in object-oriented Perl. This provides a convenient environment for the string manipulations required by the evolutionary operations and simple access to the underlying operating system (Linux). Chromosome fitness evaluation is the computationally intensive part of the evolutionary process and we currently farm this job out to a separate process running a commercial image processing engine (interactive data language (IDL), by Research Systems, Inc. [21]). IDL does not provide all the image processing operators we want, so we have implemented additional operators in $\mathrm{C}$ that can be called from within the IDL environment. Within IDL, individual genes correspond to single primitive image operators, which are coded as IDL procedures; a chromosome is a sequence of genes and exists as lines of IDL code in an IDL batch executable. In our present implementation, an IDL session is opened at the start of a run and communicates with the Perl code via a two-way UNIX pipe. This pipe is a low-bandwidth connection. It is only the IDL session that needs to access the input and training data (possibly hundreds of megabytes), requiring a high-bandwidth connection. The ALADDIN training data mark-up tool was written in Java. Fig. 2 shows the software architecture of the system.

\section{CONVENTIONAL SUPERVISED CLASSIFICATION}

Many implementations of standard supervised classifiers exist. One of the most widely used remote-sensing software packages is the ENvironment for Visualizing Imagery (ENVI) [1], which is built on IDL and is also distributed by Research Systems, Inc. Supervised classification techniques provided as 
part of the ENVI package were used in the comparison experiments with GENIE. Currently GENIE is set up to be trained using effectively three classes: "feature," "nonfeature," and "don't care" and to be able to classify every pixel in its input data into one of two classes: "feature" and "nonfeature." The normal mode of operation of the ENVI supervised classifiers is to use training data for the one "true" class, i.e., the feature of interest. The ENVI classifier is then used to classify the input image into "feature" or "unclassified". The user adjusts the parameters of the particular supervised classifier in order to attain optimal performance, with respect to feature identification. For our experiments, these parameters were adjusted to maximize the fitness defined in (1).

The one exception to this is the maximum likelihood classifier, which requires more than one class in the training data. In this case we used the "feature" and "nonfeature" classes and the maximum likelihood classifier classified every pixel in the input data into one or other of these two classes, with no "unclassified" pixels being allowed. For applying the ENVI-supplied classifiers to out-of-training-sample data, the training data (reference spectra) used in the training was provided, together with the parameters that gave optimal performance on the training data. For the GENIE case, it was simply a case of applying the algorithms found by GENIE to the out-of-training-sample data (including the linear discriminant and threshold found during training).

In Section VI, we show auxiliary results from training the ENVI classifiers with more than just these two ("feature" and "nonfeature") classes.

The following ENVI-supplied supervised classification techniques were used in the comparison experiments [22].

\section{A. (MIN) Minimum Distance}

The minimum distance supervised classification technique [22], [23] computes the mean pixel vector of the "feature" class, and then assigns new pixels to the "feature" class based on the Euclidean distance from that pixel to the mean. For the multiclass case, the pixel is assigned to the feature whose mean value is the minimum distance from the pixel. For the simple feature/nonfeature discrimination here, the pixels is identified as a "feature" if the distance is less than a user-defined threshold (adjusted to obtain optimum performance on the training data); otherwise, it is a "nonfeature."

\section{B. (MAX) Maximum Likelihood}

Maximum likelihood classification is the most common supervised classification method used with remote sensing data [23], and among the classifiers considered here, the one with the most free parameters. Here each class ("feature" and "nonfeature") is modeled with separate multivariate gaussian distributions. New pixels are assigned to the class that had the highest probability of generating that pixel.

\section{C. (MAH) Mahalanobis Distance}

The Mahalanobis distance technique [23] is very similar to the maximum likelihood classifier, but with the simplification that all classes are modeled as having identical covariance matrices (which define the shape and orientation of the normal distribution). In the one class case, we compare the probability that a new pixel was generated by the "feature" class, to a user-defined threshold, in order to decide the class to which each pixel belongs.

\section{D. (SAM) Spectral Angle Mapper}

The spectral angle mapper (SAM) technique [24] is motivated by the observation that changes in illumination caused by shadows, slope variation, sun position, light cloud, etc., approximately only alter the magnitude of a pixel's vector, rather than the direction. Therefore we can eliminate these effects by normalizing all pixel vectors to unit magnitude and then looking at the angle between a given pixel and the mean vector for the "feature" class. Pixels are assigned to the "feature" class if this angle is less than a user-defined threshold.

\section{E. (BIN) Binary Encoding}

Binary encoding classification [23], [25] encodes the data and reference spectra into ones and zeros, based on whether a particular band value lies above or below the spectrum mean. The comparison between the encoded reference spectrum with the encoded data spectra is performed using a Boolean logic exclusive $\mathrm{OR}$ (XOR) function. A user specifies the minimum fraction of bands that must match between the encoded reference spectrum and the data spectra. Pixels that do not meet this criterion are labeled as "nonfeature." We note that binary encoding produces an extreme coarsening of the data. It was invented for, and is most appropriately applied to, hyperspectral data.

It is worth noting that for the traditional supervised classifiers, the user-defined thresholds determined as being optimal for the training data may not be optimal for out-of-training-sample data. However, we can envisage a production scenario, where the classifiers are trained on one set of data to find a particular feature, where some kind of "ground truth" is available and the resultant classifier is applied to some other out-of-training-sample data, in order to determine if that particular feature is present or not in the data, and "ground truth" data not be available for that data. In this case, the lack of ground truth means that there is no quantitative way of determining the optimal threshold value for the out-of-training-sample data. It should also be pointed out that this is also the case for the GENIE classifiers. GENIEs backend has a threshold which needs to be determined and the value determined as optimal for a training set may not be optimal for out-of-training-sample data. So, for a fair comparison, thresholds determined for all classifiers during training where left unchanged when the classifiers were applied to out-of-training-sample data. In addition, experiments were also conducted in which user-adjusted thresholds were not employed, where the traditional classifiers were forced to classify the entire scene into feature or nonfeature based on the particular distance measure appropriate to the classifier. This amounts to a planar separating surface compared to a sphere for the user-defined threshold case. It was found that the user-adjusted threshold scenario performed better, in general. 
TABLE II

LIST OF DATA SETS USED IN THE EXPERIMENTS

\begin{tabular}{l|c|c}
\hline Feature & Site Name & AVIRIS Flight Number \\
\hline Roads 1 & Moffet Field & f970620t01p02_r03_sc01 \\
Roads 2 & ARM Site & f970801t01p02_r01_sc06 \\
Roads 3 & Denver & f970701t01p02_r07_sc07 \\
\hline Golf 1 & Moffet Field & f970620t01p02_r03_sc01 \\
Golf 2 & Denver & f970701t01p02_r07_sc01 \\
Golf 3 & Kennedy Space & f960323t01p02_r04_sc02 \\
& Center & \\
\hline Urban 1 & Denver & f970701t01p02_r07_sc09 \\
Urban 2 & Denver & f970701t01p02_r07_sc04 \\
Urban 3 & Moffet Field & f970620t01p02_r03_sc01 \\
Clouds 1 & Atlanta & f970806t01p02_r07_sc01 \\
Clouds 2 & Atlanta & f970806t01p02_r07_sc02 \\
Clouds 3 & "Clouds 1" & f970817t01p02_s05_sc01 \\
\hline
\end{tabular}

\section{EXPERIMENTAL DATA AND CLASSIFICATION TASKS}

\section{A. Data Used in the Experiments}

The remotely-sensed images referred to in this paper were derived from the Airborne Visible and InfraRed Imaging Spectrometer(AVIRIS) [26], a sensor developed and operated by the NASA JetPropulsion Laboratory. The AVIRIS sensor collects datain 224 contiguous, relatively narrow $(10 \mathrm{~nm})$, uniformly-spaced spectral channels. AVIRIS is an airborne sensor and spatial resolution can vary from a few meters to $20 \mathrm{~m}$, depending on the altitude of the collecting platform. We used data from 1996 and 1997 AVIRIS campaigns from a range of sites shown in Table II; more detail is available from the AVIRIS quicklook website [27].

For the studies reported here, we used a reduced number of relatively wide spectral bands, designed to simulate imagery from a new remote sensing satellite called the Multispectral Thermal Imager (MTI) [28]. The MTI satellite was launched in March 2000 and collects data in 15 spectral bands. Ten of these bands sample wavelengths between 0.4 and 2.4 microns, a region covered by the AVIRIS instrument. As test data to develop analysis codes for the MTI mission, AVIRIS data were convolved with the MTI spectral filter functions to produce simulated MTI data. This 10-band simulated data was used for development of both conventional remote sensing algorithms and for GENIE development, such as reported here.

The images displayed here are false-color images (which have then been converted to gray-scale in the printing process). The color mappings used are the same for all original image data shown. The particular color mappings used here involve averaging MTI bands A $(0.45-0.52 \mu \mathrm{m})$ and B $(0.52-0.60$ $\mu \mathrm{m})$ for the blue component, bands $\mathrm{C}(0.62-0.68 \mu \mathrm{m})$ and $\mathrm{D}(0.76-0.86 \mu \mathrm{m})$ for the green component and bands $\mathrm{E}$ $(0.86-0.89 \mu \mathrm{m})$ and $\mathrm{F}(0.91-0.97 \mu \mathrm{m})$ for the red component. In addition, the images have been contrast enhanced. The choice of color mappings was arbitrary, in that it was a personal decision made by the analyst in order to best "highlight" the feature of interest, and thereby enable the production of high quality training data. This ability to manipulate the image with color mappings and contrast enhancement is an important feature of the graphical interface.

\section{B. Classification Tasks}

We chose four different features of interest: roads, golf courses, urban areas, and clouds. These features were chosen
TABLE III

COMPARISON OF GENIES EVOLVED ALGORITHM WITH ENVI ALGORITHMS $(\mathrm{DR}=$ DETECTION RATE, FAR $=$ FALSE ALARM RATE $)$

\begin{tabular}{|c|c|c|c|c|c|c|c|c|c|c|c|c|c|}
\hline & \multicolumn{6}{|c|}{ Treining Data } & \multicolumn{6}{|c|}{ Out-of-Training-Sample Data } \\
\hline & & GENIE & MIN & & & SAM & BIN & GENIE & & MAH & & & BIN \\
\hline \multirow{4}{*}{ Roads } & Fitness & 963.3 & 781.2 & 865.9 & 921.1 & 803.9 & 677.5 & 763.2 & 559.9 & \begin{tabular}{|l|l|}
500.0 \\
\end{tabular} & 611.2 & 566.0 & 587.1 \\
\hline & $\mathrm{DR} 0^{\circ}$ & 96.61 & 78.63 & 83.11 & 91.62 & 82.93 & 82.00 & 60.71 & 27.54 & 0.00 & 62.25 & 30.29 & 72.94 \\
\hline & FAR & 3.95 & 22.40 & 10.03 & 70 & 2214 & 46 & 736 & & & & 1709 & \\
\hline & Rank & $1 \mathrm{st}$ & 5 th & $3 r d$ & 2 nd & 4 th & 6th & lst & & 6th & 2nd & th & 3rd \\
\hline \multirow{4}{*}{ Golf } & Fitness & 998.3 & $9 \overline{45.0}$ & 947.6 & 966.2 & 915.4 & 820.1 & 739.8 & 584.8 & 500.0 & 553,1 & 696.5 & $\overline{572.9}$ \\
\hline & DR $(\%)$ & 99.68 & 95.16 & 93.84 & 96.21 & 92.73 & 78.72 & 61.60 & 42.34 & 0.00 & 10.93 & 58.76 & 51.16 \\
\hline & FAR (\%) & 0.00 & 6.17 & 4.32 & 2.97 & 9.64 & 14.71 & 13.65 & 25.39 & 0.00 & 0.31 & 19.46 & 36.57 \\
\hline & Rank & lat & thth & $3 \mathrm{rd}$ & 2nd & 5 th & 6 th & list & $\begin{array}{l}20.03 \\
3 \mathrm{rd}\end{array}$ & 6 th & 5 th & 2nd & $\begin{array}{l}\text { ath } \\
\text { Ath }\end{array}$ \\
\hline \multirow{4}{*}{ Urban } & Fitne & $\overline{998.9}$ & 6994.7 & $\overline{861.6}$ & 963.6 & 636.3 & $\overline{580}$ & $\overline{813.5}$ & {$[58 \overline{6.2}$} & $5 \overline{14.6}$ & 569.4 & $\overline{499.1}$ & 521.9 \\
\hline & $\mathrm{DR}(\%)$ & 99.85 & 58.55 & 80.34 & 95.67 & 75.03 & 83.59 & 66.32 & 27.36 & 2.93 & 65.86 & 50.67 & 70.18 \\
\hline & FAR (\%) & 0.07 & 19.61 & 8.03 & 2.94 & 47.77 & 67.32 & 3.63 & 10.11 & 0.02 & 51.97 & 50.51 & 65.80 \\
\hline & Rank & 1st & 4th & 3rd & 2nd & 5 th & 6 th & lst & 2nd & 5 th & $3 \mathrm{rd}$ & 6th & 4th \\
\hline \multirow{4}{*}{ Clouds } & Fitne & 9999.9 & & 946.7 & & 979.4 & $\overline{760}$ & 978.0 & 968.6 & & 701.7 & 975.3 & 727.1 \\
\hline & $\mathrm{DR}(\%)$ & 99.99 & 96.41 & 94.21 & 99.91 & 98.18 & 55.59 & 97,43 & 95.38 & 28.62 & 99.97 & 97.28 & 48.85 \\
\hline & FAR & 0.00 & 1.27 & 4.86 & 0.33 & 2.29 & 3.5 & 1.82 & & 2.22 & 59.64 & 2.23 & 3.43 \\
\hline & Rank & lst & 4th & 5 th & 2nd & $3 \mathrm{rd}$ & 6 th & $1 \mathrm{st}$ & 3rd & 6 th & $5 \mathrm{sh}$ & 2nd & 4th \\
\hline \multirow{4}{*}{ Average } & Fitne: & & & 905.5 & 12 & 833.8 & 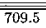 & 82 & .9 & 536.6 & 608.8 & 4.2 & 3 \\
\hline & & & 82.19 & & 95.86 & 87.22 & 74. & 71.51 & 48.16 & & 59.75 & 59.25 & 60.78 \\
\hline & FAR & $1.0 \mathrm{E}$ & 12.36 & 6.81 & 3.41 & 20.46 & 33.07 & 6.62 & $\begin{array}{l}43.18 \\
13.18\end{array}$ & 0.56 & 37.99 & 22.32 & 4033 \\
\hline & Rank & lst & th & $3+d$ & $2 n d$ & 5 th & 6 th & 1 st & $3 \mathrm{rd}$ & 6 th & $4 t h$ & 2nd & 5 th \\
\hline
\end{tabular}

because of their particular attributes in multispectral data. The features were considered a good test of a supervised classification technique due to the different levels of difficulty they posed for these techniques. Clouds are relatively easy, and mostly spectral; urban areas encompass a land-cover distinction; roads are easy for the eye to find, but notoriously difficult for automated algorithms; golf courses require a combination of spectral and spatial information to disambiguate them from other similarly-vegetated areas (e.g., lawns).

We set the various supervised classification techniques the task of distinguishing these features within several scenes of the ten-channel multispectral data as described above. For each feature of interest three separate scenes had training data marked-up using the ALADDIN tool. This provided "ground truth" for training data and for assessing the performance of the classification scheme on out-of-training-sample data. We employed a cross-validation scheme where, for each feature, we trained a classifier separately on the three marked-up scenes, and then for each scene, applied the resulting classifier to the two remaining out-of-sample scenes. GENIE was run, with a population of 100 individuals, for 500 generations, or until a (perfect score) fitness of 1000 was achieved.

An example of an image plus associated training data is shown in Fig. 1. This figure shows the false-color image for one of the scenes used for the "urban area" feature classification, and the associated training data. In the training data image the white pixels correspond to the places on the image where the feature is asserted to be, the grey pixels to where the feature is asserted not to be, and the black pixels correspond to places where no assertion is made.

\section{COMPARISON EXPERIMENTS}

For the training phase, we ran GENIE and the ENVI-supplied classifiers on the training data. For GENIE, the result of this training phase is an image processing pipeline which can be applied to and tested on other data. To apply the ENVI-supplied classifiers to out-of-training-sample data it was necessary to save the regions of interest of the marked-up training classes and provide them as the reference spectra for application of the classifiers to out-of-training-sample data.

We measured the fitness, detection rate and false-alarm rate of all the classifiers on the training data and out-of-training-sample 


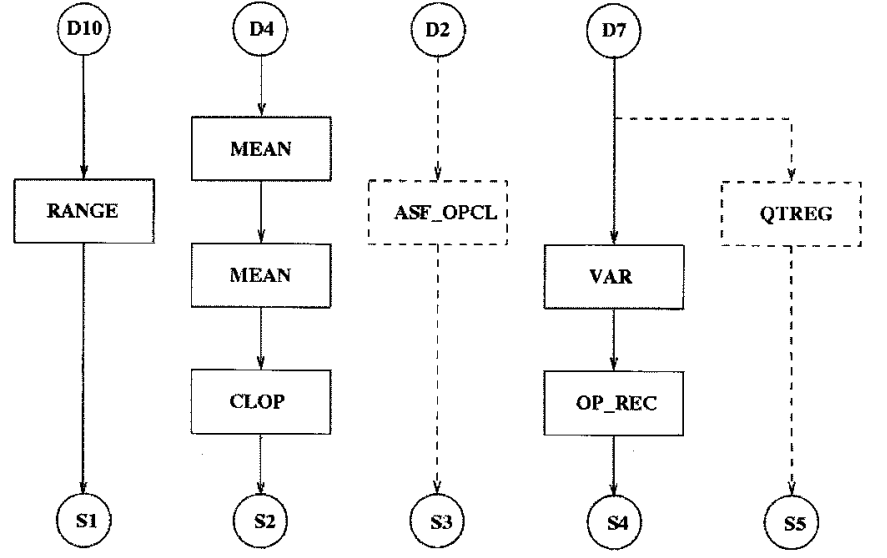

Fig. 3. Image processing pipeline discovered by GENIE for finding golf courses. Dotted lines indicate scratch planes which did not contribute significantly to the final classification.

data. Table III summarizes the quantitative results of the comparison between the GENIE algorithm output and the traditional algorithms' output for each of the features. The bottom four rows of the table show the average, for each classification technique, across all features sought. It is interesting to notice that the relative ranking (based on fitness score) of each of the classifiers is relatively stable over the different features, with the more complicated classifiers generally achieving the highest scores. For the out-of-training-sample data, by contrast, the simpler algorithms (with fewer free parameters) perform much better. The main exception is GENIE, which performs well on both the traning data and on the out-of-training-sample data.

An example of an image processing pipeline produced by GENIE is given by the following solution to the golf coursefinding task:

\begin{tabular}{|c|c|}
\hline [QTREG $\quad$ rD7 $\quad$ wS5 $\quad$ wS3 wS1 & $0.05] *$ \\
\hline$\left[\begin{array}{lllll}\text { MEAN } & \text { rD4 } & \text { wS2 } & 4 & 0\end{array}\right]$ & \\
\hline$\left[\begin{array}{lllll}\text { MEAN } & \text { rS2 } & \text { wS2 } & 3 & 0\end{array}\right]$ & \\
\hline$\left[\begin{array}{lllll}\text { VAR } & \text { rD7 } & \text { wS4 } & 3 & 0\end{array}\right]$ & \\
\hline$\left[\begin{array}{lllll}\text { CLOP } & \text { rS2 } & \text { wS2 } & 3 & 0\end{array}\right]$ & \\
\hline$\left[\begin{array}{lllll}\text { RANGE } & \text { rD10 } & \text { wS1 } & 3 & 0\end{array}\right]$ & \\
\hline$\left[\begin{array}{lllll}\mathrm{OP} \perp \mathrm{REC} & \mathrm{rS} 4 & \mathrm{wS} 4 & 3 & 0\end{array}\right]$ & \\
\hline$[$ ASF_OPCL $\quad$ rD2 $\quad$ wS3 310$] *$ & \\
\hline
\end{tabular}

As described in Section II-B, each line consists of a single primitive image processing operation: the name of the operator, which data (D) or scratch (S) planes were read ( $r$ ) from and which were written (w) to, and what parameter values were used (see Table I for details on the individual operators). GENIE produced a solution with five answer planes, and the backend produced a linear combination of those planes, along with a threshold value, to give a binary classification. A graphical representation of this pipeline is illustrated in Fig. 3. Note that the circled Ds represent the input data planes and the circled $\mathbf{S s}$ represent the answer planes that are input to the back-end classifier (Fisher Linear Discriminant plus threshold), to produce the final classification result. To aid clarity, we now provide a narrative description of the operation of this pipeline.
The RANGE operator computes the difference between the maximum and minimum value in a $7 \times 7$ kernel of data plane D10, and writes the result to scratch plane $\mathrm{S} 1$. The parameters " 30 " correspond to a square $7 \times 7$ kernel. The first integer parameter for this operator, "3," actually defines the "radius" of the smoothing kernel, where the "diameter" of the kernel is always an odd integer, and defined as $(2 \times$ radius $)+1$. The second integer parameter, " 0 ", defines the particular choice of kernel shape, in this case a square. A " 1 " would define a circle, "2" a vertical cross, "3" a diagonal cross, etc.

The first MEAN operator, [MEAN rD4 wS2 4 0], smooths the data plane D4 with a $9 \times 9$ square kernel, and writes the solution to scratch plane $\mathrm{S} 2$. The second MEAN operator, [MEAN rS2 wS2 3 0], smooths the result stored in the S2 plane with a $7 \times 7$ square kernel, and the CLOP operator performs a morphological close-open operation, again with a $7 \times 7$ square kernel, writing the output to scratch plane S2.

The ASF_OPCL operator performs an alternating sequential open-closing with a square kernel of maximum size $7 \times 7$ on data plane D2, and writes the output to scratch plane S3.

The VAR operator computes the variance in a $7 \times 7 \mathrm{kernel}$ of data plane D7, and writes the result to scratch plane S4. That plane is further modified by the OP_REC operator, which performs a morphological opening with reconstruction, again based on a $7 \times 7$ kernel.

The QTREG operator also reads data plane D7 and writes three scratch planes (S5, S3, and S1), two of which (S3 and S1) are overwritten by other operators before being used.

Finally, the Fisher Discriminant backend applies a linear combination of the scratch planes, followed by a threshold, to produce a binary answer plane. The coefficients applied to the five answer planes (SO, S1, S2, S3, S4) are: $\{-9.354 \times$ $\left.10^{-6}, 1.235 \times 10^{-5}, 1.659 \times 10^{-6}, 1.460 \times 10^{-9}, 0.0349\right\}$. There is an additional dc offset value of -0.350 applied to the output of the linear combination. The threshold value for determining the binary output was 0.664305 .

It can be seen that this image processing pipeline has only used four of the available ten data planes as input: data planes D2, D4, D7, and D10. These correspond to the MTI bands B $(0.52-0.60 \mu \mathrm{m}), \mathrm{D}(0.76-0.86 \mu \mathrm{m}), \mathrm{G}(0.99-1.04 \mu \mathrm{m})$, and O $(2.08-2.35 \mu \mathrm{m})$, respectively. GENIEs choice of input data bands is (in retrospect) not too surprising, given the task. The algorithm is using the green band (B), as well as two near-infrared (NIR) bands (D,G) and a short-wave infrared (SWIR) band (O). Vegetation is highlighted in the two NIR bands that GENIE selected, as well as in the green band.

Of these five answer planes the most important were S1, S2, and S4; using only those planes we could still achieve the same fitness value, on the training data and out-of-training-sample data, as when all the answer planes were used. Hence, two of the operators did not contribute substantially to the solution. The outputs of the useful answer planes, as can be seen from Fig. 3, are derived from the NIR and SWIR bands. In this case we see, somewhat surprisingly, that the green band is not contributing significantly to the solution. We might expect green to be very useful for identifying golf courses, and this is probably how it made its way into the chromosome. However, in the end, the NIR and SWIR bands were found to be more informative. 


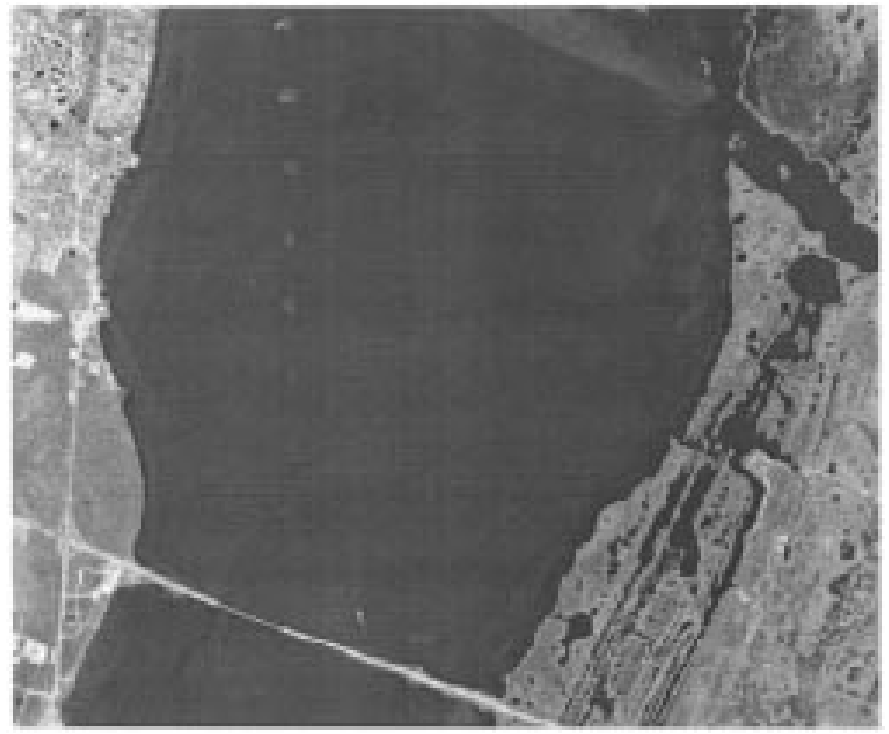

(a)

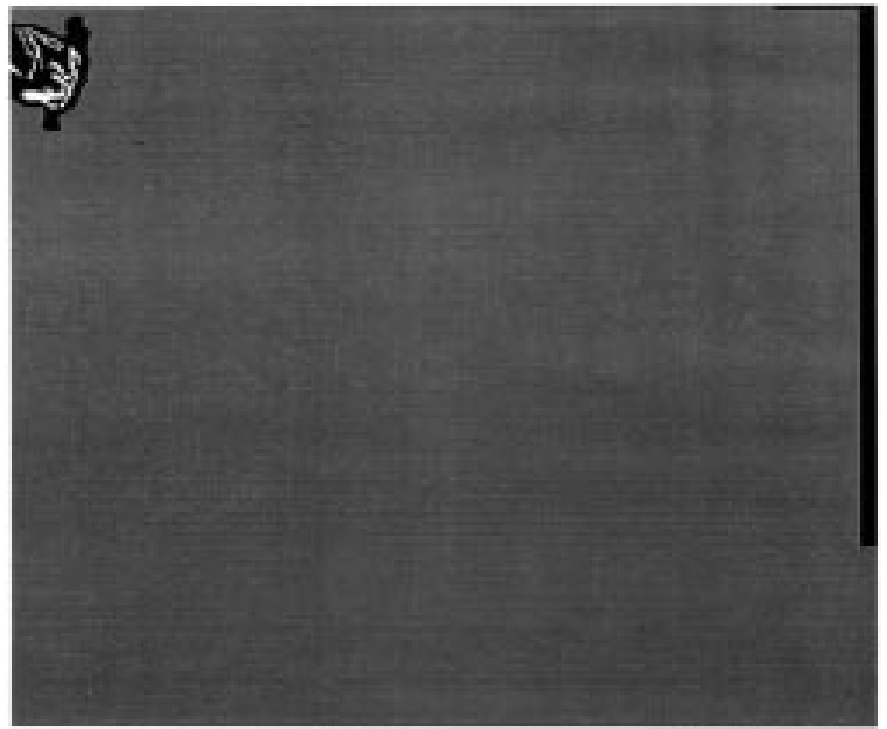

(b)

Fig. 4. (a) Greyscale images of one of the scenes used to produce the training data for "Golf Courses" (Golf 3). (b) Training data provided for the training scene for "Golf Courses" (white = feature, grey = not feature, and black = no assertion). The black "buffer area" around the golf course reflects the analyst's lack of concern with a detailed delineation of the precise extent of the golf course.

We illustrate the results of these classification techniques on training and out-of-training-sample data with an example of output from GENIE, and from the best-performing ENVI classifier, on the golf course problem. Figs. 4 and 5 compare GENIE to MAX for one of the training data sets, and Figs. 6 and 7 compare GENIE to the Spectral Angle Mapper (SAM) on out-oftraining-sample data.

An interesting aspect of GENIEs performance to consider is its repeatability; i.e., whether or not, for a given feature, GENIE leads to the same result (i.e., the same "image processing pipeline") when trained on different scenes. In general, GENIE will not produce the same image processing pipeline even when trained on the same scene, if it starts with a different random number seed. However, the different solutions will generally

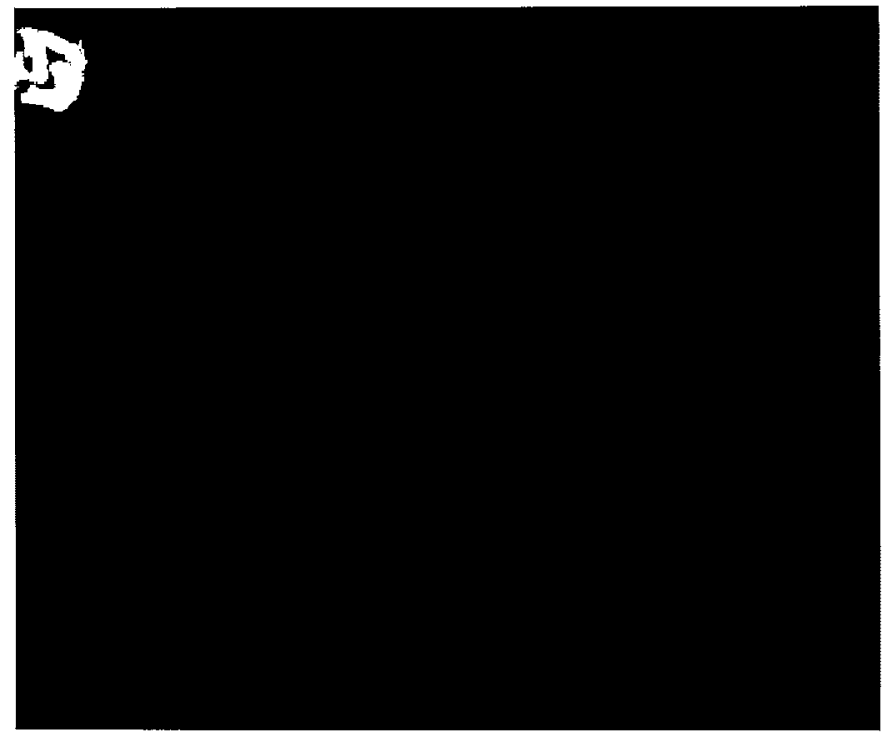

(a)

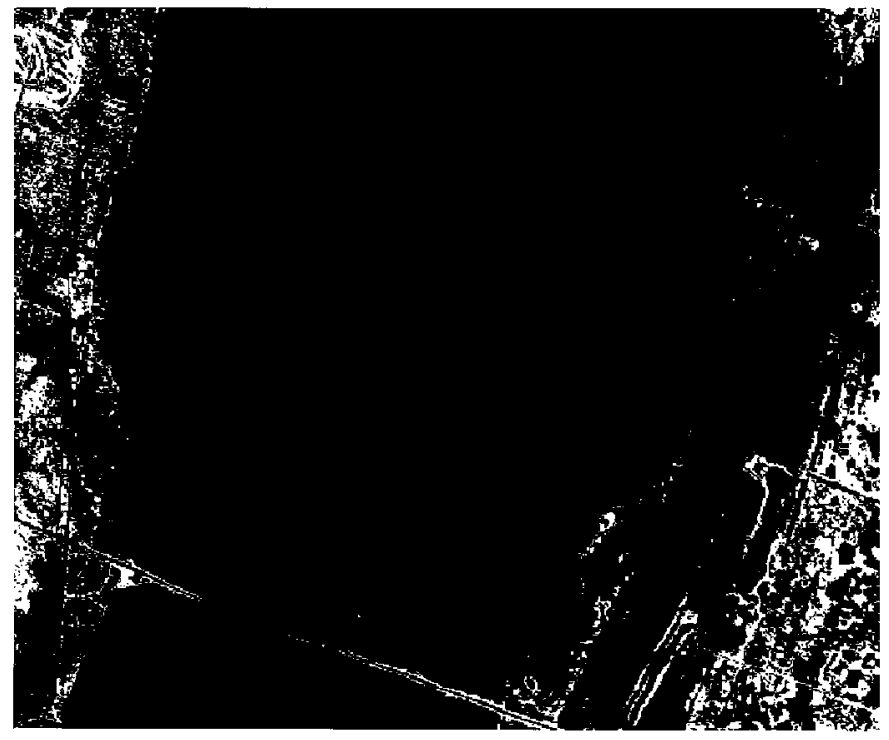

(b)

Fig. 5. (a) GENIE results on training data: Fitness $=$ 999.2. (b) Best ENVI classifier for the particular training scene (minimum distance): Fitness $=957.4$. Here, GENIEs use of spatial information is clearly evident. The ENVI classifier actually did a better job of delineating the extent of the golf course, whereas GENIEs spatial operators led to a "fatter" golf course than the purely spectral data would warrant. On the other hand, though, this spatial information allowed GENIE to veto the golf course-like spectra in the rest of the image. Because the "fatter" golf course fits inside the no-assertion region, GENIE is not penalized.

have the same approximate performance, both on training data and on out-of-sample data, and there will often be an overlap in the choice of operators and data planes used in the image processing pipeline that is evolved. But the space of image processing pipelines it too large and too rugged to achieve any real level of "robustness," in this regard.

\section{FURTHER EXPERIMENTS AND RESULTS}

Depending on the application at hand, an image analyst is sometimes interested in the identification of a single specific feature against a background of everything else in the image, 


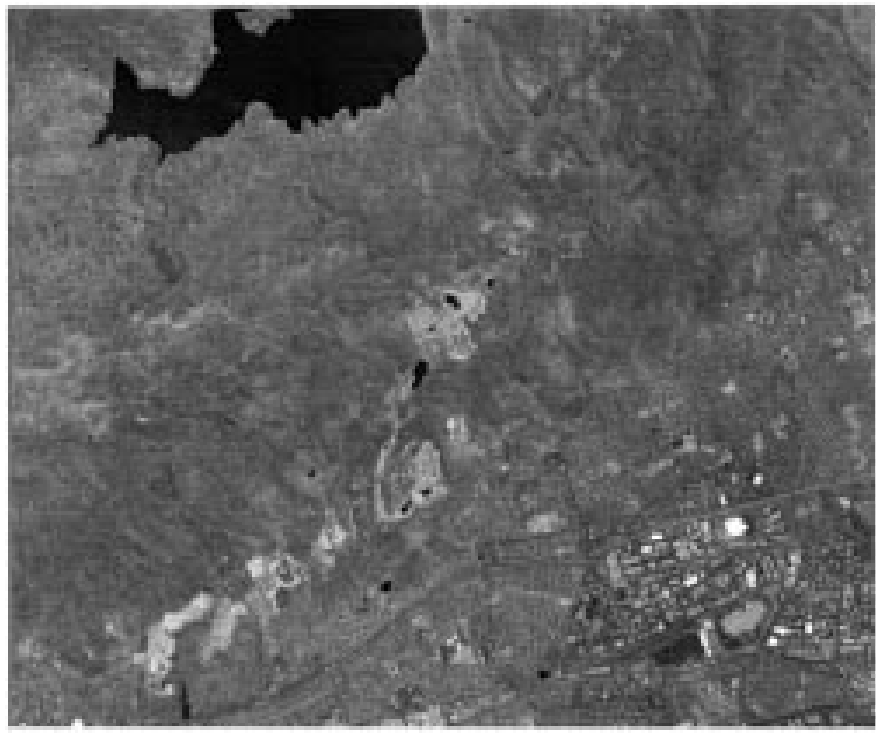

(a)

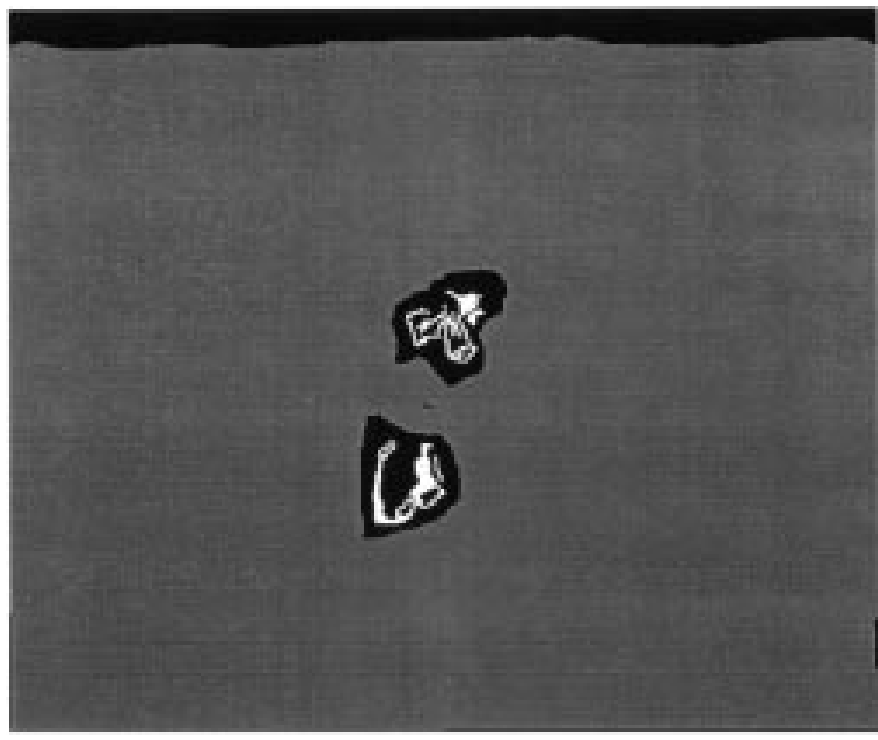

(b)

Fig. 6. (a) Greyscale images of one of the scenes used to produce training data for "Golf Courses" (Golf 1). (b) Training data provided for the training scene for "Golf Courses" (white $=$ feature, grey $=$ not feature, and black $=$ no assertion).

and is sometimes interested in the simultaneous extraction of multiple features (for instance, when making a landcover map). The experiments described in the previous sections take the first point of view and it is this binary classification task that GENIE was designed to handle.

However, for MAX and other conventional classifiers, the "background of everything else" is not well modeled as a single unimodal class. To address this difficulty, it has been suggested [29] to artificially divide the background into multiple classes, and then employ multi-class classification techniques. This combined use of labeled and unlabeled samples can often lead to more powerful supervised classification [30]-[33].

In order to address these same issues, we conducted a series of further experiments where we adopted a similar approach, in which the standard supervised classification techniques were

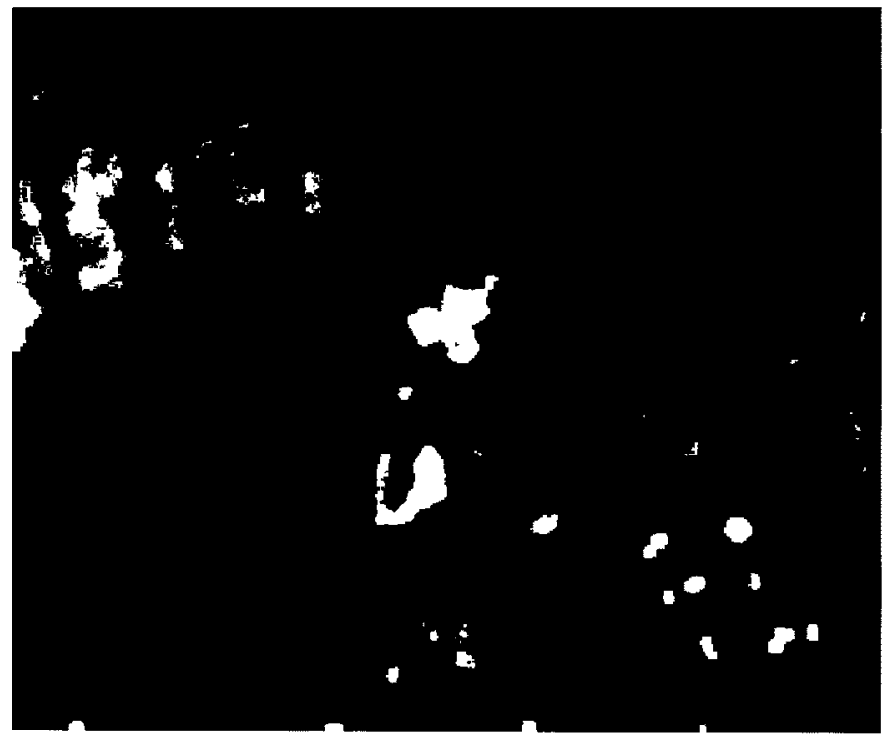

(a)

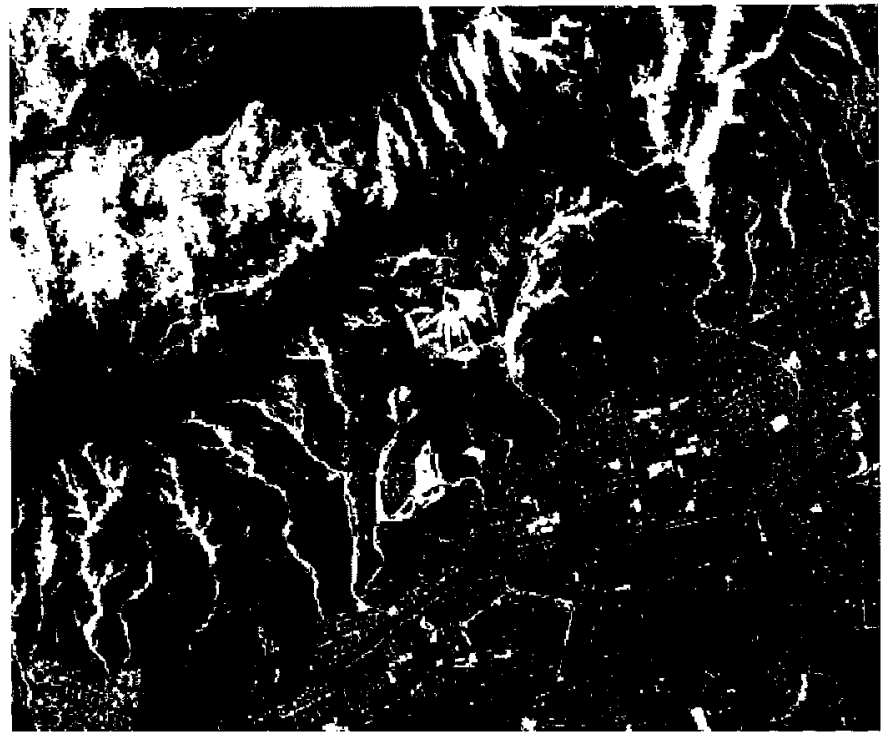

(b)

Fig. 7. (a) GENIE results on out-of-training-sample data: Fitness $=946.9$. (b) Best ENVI classifier (for particular training scene) on out-of-training-sample data (spectral angle mapper): Fitness $=856.7$. Again, Genie has used its spatial operators to produce "fatter" golf courses, but it was also able to censor more of the nongolf-course area in the rest of the scene.

given the task of classifiying the scenes into multiple classes instead of the two feature/nonfeature classes described in the previous experiments.

\section{A. Experimental Procedure}

The training data as provided to GENIE and as used in the experiments described in Section $\mathrm{V}$ were used to create the training data provided to the standard supervised classifiers. The "feature" class was kept as it was, but the "nonfeature" class was divided up into multiple classes. The combination of the "feature" class and the subdivided "nonfeature" class was then given as training data to the standard supervised classification techniques. 
TABLE IV

RANKINGS, BASED on Fitness SCORES AVERAGEd OvER ALl ClasSifiCATION TASKS, OF THE BEST MULTIPLE-Class VARIANTS OF THE STANDARD SUPERVISED CLASSIFIERS; ON BOTH TRAINING DATA AND TESTING DATA

\begin{tabular}{l||c|c||c|c}
\hline \multicolumn{1}{l||}{} & \multicolumn{2}{c||}{ Training } & \multicolumn{2}{c}{ Out-of-Training-Sample } \\
\hline Rank & Classifier & Fitness & Classifier & Fitness \\
\hline 1st & GENIE & 990.1 & GENIE & 824.5 \\
\hline 2nd & MAX (M) & 965.8 & SAM (M) & 780.0 \\
\hline 3rd & MAH (M) & 949.1 & MIN (M) & 778.7 \\
\hline 4th & MAH & 905.5 & MAH (M) & 752.4 \\
\hline 5th & MIN (M) & 892.3 & SAM & 684.2 \\
\hline 6th & SAM (M) & 874.1 & MIN & 674.9 \\
\hline 7th & MIN & 849.1 & MAX (M) & 615.5 \\
\hline 8th & SAM & 833.8 & MAH (M) & 536.6 \\
\hline
\end{tabular}

It should be noted that the binary encoding supervised classification technique was not included in these additional experiments.

The "nonfeature" class was divided into multiple classes by applying ENVIs unsupervised k-means classification algorithms [1], [34], [35], to the entire "nonfeature" class. This k-means classification was performed several times, varying the number of classes into which the nonfeature class was classified. The $\mathrm{k}$-means classification with the number of classes that provided the best final classification performance in terms of fitness, was the one included in the additional results shown here.

Table IV shows the overall ranking for the multiple-class classification algorithms, averaged over all the features for the training data. In this table, "(M)" indicates the use of multi-class training; the nonmultiple-class results are those results described and shown in Section V.

\section{DISCUSSION}

With a single exception, GENIE outperformed all the other classification techniques on both training data and out-of-training-sample data, for all of the classification tasks considered. For the training data, the gap, with respect to fitness, between GENIEs performance and the best of the other techniques was much less than for the out-of-training-sample case. This suggests that GENIE is significantly better at generalizing than the other techniques compared here. An interesting observation is that the best of the other techniques on the training data did not necessarily guarantee it to be the best of the other techniques on the out-of-training-sample data. This indicates the sensitivity of these techniques to training data and highlights GENIEs generalization abilities.

The one exception was the multiclass SAM applied to golf courses, on out-of-training-sample data. This suggests that golf courses are relatively well identified by their spectral signatures (perhaps not surprising in a desert/mountain environment where they are quite distinctive), and that the illumination-invariance built into the SAM provided it the edge to better generalize to other scenes. Since GENIE was trained on only one scene at a time, it did not "learn" to employ an illumination-independent solution.

One issue to be addressed is training time. At present GENIE requires the testing of potentially thousands of candidate algorithms on the training data. Depending on the size of the data, this can take hours to complete. This is considerably longer to train than the other techniques. It should be noted, though, that the result of GENIEs training is an image processing algorithm that can be applied to other data with times comparable to that of the other techniques' application to out-of-training-sample data. We also remark that a few hours is usually a small fraction of the time it would take to hand-design an equivalent image processing pipeline that is customized not only to the specific feature, but also to the specific data set. Another point to consider is that being a population-based optimization technique, GENIE lends itself well to parallelization, which can dramatically reduce training time. Some experiments have been carried out to demonstrate this [6].

Although the traditional classification techniques that were compared here use only spectral information, it is possible to enable these techniques to use spatial information as well. There is in fact a large literature on methodologies for combining spatial and spectral information (e.g., see [36]-[39]). Our approach was to apply a set of spatial operators to each plane in the input multispectral data and then combine these new processed data planes with the raw data planes; both sets of planes would then be provided as input to the supervised classifiers. We applied a number of morphological smoothings at different scales to the input data and combined this with the original data. We found that this information did improve the fitness scores achieved by the conventional supervised classifiers, but they were still considerably below the performance of GENIE on the original data. Also, the improved performance was only for the training data. The classifiers actually performed worse on out-of-training-sample data (i.e., they were less robust). Obviously, if one were to adopt this approach, the choice of which spatial operators to apply is very important and the search space in this regard is immense. If one considers a scenario where some sophisticated technique is used to search the space for the optimal combination of spatial operators, one is entering the arena in which GENIE is designed to function.

In conclusion, an automated feature detection/classification system based on genetic programming has been described. Experiments comparing this new system with traditional supervised classifiers indicate consistently better performance, on both training data and out-of-training-sample data. We attribute GENIEs success to the choice of solution representation - as a multispectral image processing pipeline-and to the fact that it very naturally combines information from both the spectral and spatial domains.

\section{REFERENCES}

[1] Envi-RSI's Remote Sensing Software [Online]. Available: http://www.rsinc.com/envi/index.cfm

[2] S. P. Brumby, J. Theiler, S. J. Perkins, N. R. Harvey, J. J. Szymanski, J. J. Bloch, and M. Mitchell, "Investigation of image feature extraction by a genetic algorithm," Proc. SPIE, vol. 3812, pp. 24-31, 1999.

[3] J. Theiler, N. R. Harvey, S. P. Brumby, J. J. Szymanski, S. Alferink, S. Perkins, R. Porter, and J. J. Bloch, "Evolving retrieval algorithms with a genetic programming scheme," Proc. SPIE, vol. 3753, pp. 416-425, 1999.

[4] N. R. Harvey, S. Perkins, S. P. Brumby, J. Theiler, R. B. Porter, A. C. Young, A. K. Varghese, J. J. Szymanski, and J. J. Bloch, "Finding golf courses: The ultra high tech approach," in Lecture Notes in Computer Science, 2000, vol. 1803, pp. 54-64.

[5] S. P. Brumby, J. Theiler, N. R. Harvey, S. J. Perkins, R. B. Porter, J. J. Szymanski, and J. J. Bloch, "A genetic algorithm for combining new and existing tools for multispectral imagery," Proc. SPIE, vol. 4049, 2000. 
[6] N. R. Harvey, S. P. Brumby, S. J. Perkins, R. B. Porter, J. Theiler, A C. Young, J. J. Szymanski, and J. J. Bloch, "Parallel evolution of image processing tools for multispectral imagery," Proc. SPIE, vol. 4132, pp. $72-82,2000$.

[7] K. L. Hirsch, S. P. Brumby, N. R. Harvey, and A. B. Davis, "The MTI dense-cloud mask algorithm compared to a cloud mask evolved by a genetic algorithm and to the MODIS cloud mask," Proc. SPIE, vol. 4132 2000.

[8] S. J. Perkins, J. Theiler, S. P. Brumby, N. R. Harvey, R. B. Porter, J. J. Szymanski, and J. J. Bloch, "GENIE: A hybrid genetic algorithm for feature classification in multispectral images," Proc. SPIE, vol. 4120, pp. 52-62, 2000.

[9] C. Harris and B. Buxton, "Evolving Edge Detectors," Dept. of Computer Science, University College London, London, U.K., Res. Note RN/96/3, 1996.

[10] N. R. Harvey and S. Marshall, "GA optimization of multidimensional grey-scale soft morphological filters with applications in archive film restoration," in Mathematical Morphology and its Applications to Image and Signal Processing V (ISMM2000), J. Goutsias, L. Vincent, and D. S. Bloomberg, Eds. Norwell, MA, pp. 129-138.

[11] A. Teller and M. Veloso, "A controlled experiment: Evolution for learning difficult image classification," in Lecture Notes in Computer Science, 1995, vol. 990, pp. 165-176.

[12] R. Poli et al., "Genetic programming with user-driven selection: Experiments on the evolution of algorithms for image enhancement," presented at the 2nd Annu. Conf. Genetic Programming, J. R. Koza et al., Eds., San Francisco, CA, 1997

[13] J. M. Daida, J. D. Hommes, T. F. Bersano Begey, S. J. Ross, and J. F Vesecky, "Algorithm discovery using the genetic programming paradigm: Extracting low-contrast curvilinear features from SAR images of arctic ice," in Advances in Genetic Programming 2, P. J. Angeline and K. E. Kinnear, Eds. Cambridge, MA: MIT Press, 1996, ch. 21.

[14] S. Bandyopadhyay and S. K. Pal, "Pixel classification using variable string genetic algorithms with chromosome differentiation," IEEE Trans. Geosci. Remote Sensing, vol. 39, pp. 303-308, Feb. 2001

[15] L. A. Cox Jr., L. Davis, and Y. Qiu, Handbook of Genetic Algorithms, L. Davis, Ed. New York, NY: Van Nostrand Reinhold, 1991, pp. 124-143. Dynamic anticipatory routing in circuit-switched telecommunications networks.

[16] T. Dandekar and P. Argos, "Potential of genetic algorithms in protein folding and protein engineering simulations," Protein Eng., vol. 5, pp. 637-645, 1992

[17] J. R. Koza, Genetic Programming: On the Programming of Computers by Means of Natural Selection (Complex Adaptive Systems). Cambridge, MA: MIT Press, 1992.

[18] K. I. Laws, "Rapid texture identification," Proc. SPIE, vol. 238, pp 376-380, 1980

[19] M. Pietikainen, A. Rosenfeld, and L. S. Davis, "Experiments with texture classification using averages of local pattern matches," IEEE Trans. Syst., Man and Cybern., vol. SMC-13, pp. 421-426, May 1983.

[20] C. M. Bishop, Neural Networks for Pattern Recognition: Oxford University Press, 1995 , ch. 3, pp. 105-112.

[21] Research Systems Software Homepage [Online]. Available: http://www.rsinc.com

[22] Envi Multispectral Classification Tutorial [Online]. Available: http://www.rsinc.com/Envi/tut2.cfm

[23] J. A. Richards and X. Jia, Remote Sensing Digital Image Analysis. New York: Springer-Verlag, 1999.

[24] F. A. Kruse, A. B. Lefkoff, J. B. Boardman, K. B. Heidebrecht, A. T. Shapiro, P. J. Barloon, and A. F. H. Goetz, "The spectral image processing system (SIPS) - Interactive visualization and analysis of imaging spectrometer data," Remote Sens. Environ., vol. 44, pp. 145-163, 1993.

[25] A. S. Mazer, M. Martin, M. Lee, and J. E. Solomon, "Image processing software for imaging spectrometry analysis," Remote Sens. Environ., vol. 24, pp. 201-210, 1988.

[26] AVIRIS Home Page [Online]. Available: http://aviris.jpl.nasa.gov

[27] AVIRIS Quicklooks [Online]. Available: http://aviris.jpl.nasa.gov/html/aviris.quicklooks.html

[28] P. G. Weber, B. C. Brock, A. J. Garrett, B. W. Smith, C. C. Borel, W. B. Clodius, S. C. Bender, R. R. Kay, and M. L. Decker, "Multispectral thermal imager mission overview," Proc. SPIE, vol. 3750, pp. 340-346, 1999.

[29] B. Jeon and D. A. Landgrebe, "Partially supervised classification using weighted unsupervised clustering," IEEE Trans. Geosci. Remote Sensing, vol. 37, pp. 1073-1079, Mar. 1999.
[30] B. Shahshahani and D. Landgrebe, "Classification of multi-spectral data by joint supervised-unsupervised learning," Sch. of Elect. Eng., Purdue Univ., West Lafayette, IN, Tech. Rep. TR-EE 94-1, 1994

[31] V. Castelli and T. M. Cover, "On the exponential value of labeled samples," Pattern Recognit. Lett., vol. 16, pp. 105-111, 1995.

[32] P.-F. Hsieh and D. Landgrebe, "Statistics enhancement in hyperspectral data analysis using spectral-spatial labeling, the EM algorithm, and the leave-one-out covariance estimator," Proc. SPIE, vol. 3438, pp. 183-190, 1999.

[33] L. Bruzzone and D. F. Prieto, "Unsupervised retraining of a maximum likelihood classifier for the analysis of multitemporal remote sensing images," IEEE Trans. Geosci. Remote Sensing, vol. 39, pp. 456-460, Feb. 2001

[34] J. T. Tou and R. C. Gonzales, Pattern Recognition Principles. Reading, MA: Addison-Wesley, 1974.

[35] A. K. Jain, M. N. Murty, and P. J. Flynn, "Data clustering: A review," ACM Comput. Surv., vol. 31, pp. 264-323, 1999.

[36] D. A. Landgrebe, "The development of a spectral-spatial classifier for earth observational data," Pattern Recognit., vol. 12, pp. 165-175, 1980

[37] B. Jeon and D. A. Landgrebe, "Classification with spatio-temporal interpixel class dependency contexts," IEEE Trans. Geosci. Remote Sensing, vol. 36, pp. 182-191, July 1992.

[38] T. Yamazaki and D. Gingras, "Image classification using spectral and spatial information based on MRF models," IEEE Trans. Image Processing, vol. 4, pp. 1333-1339, Sept. 1995.

[39] J. Theiler and G. Gisler, "A contiguity-enhanced $k$-means clustering algorithm for unsupervised multispectral image segmentation," Proc. SPIE, vol. 3159, pp. 108-118, 1997.

Neal R. Harvey received the B.Eng.(Hons.) degree in mechanical engineering from the University of Hertfordshire, U.K., in 1989, and the M.Sc. degree in information technology systems and the Ph.D. degree in nonlinear digital image processing from the University of Strathclyde, U.K. in 1992 and 1997, respectively.

He was a Research Fellow in the Signal Processing Division of the Department of Electronic and Electrical Engineering, University of Strathclyde. In April 1999, he took up a Postdoctoral Research Associate position in the Space and Remote Sensing Sciences Group of the Nonproliferation and International Security Division, Los Alamos National Laboratory, Los Alamos, NM. His research interests include nonlinear digital filters, optimization techniques, machine learning, image classification, remote sensing, and film and video restoration.

James Theiler received the Ph.D. degree from California Institute of Technology, Pasadena, in 1987, on algorithms for identifying chaos in time series.

He joined the Space and Remote Sensing Sciences Group, Los Alamos National Laboratory, Los Alamos, NM, in 1994.

Steven P. Brumby received the Ph.D. degree in theoretical particle physics from the University of Melbourne, Australia, in 1997.

He is currently a Technical Staff Member with the Space and Remote Sensing Sciences Group, Los Alamos National Laboratory, Los Alamos, NM. His research interests include automatic feature extraction, remote sensing of the environment, genetic programming, extra-galactic astrophysics, and the mathematical foundations of quantum mechanics.

Simon Perkins received the Ph.D. degree in artificial intelligence from the University of Edinburgh, U.K., in 1999.

He held a postdoctoral position at Los Alamos National Laboratory, Los Alamos, NM, from 1999 to 2001, and has been a Technical Staff Member since April 2001. His current research involves taking modern statistical machine learning methods (support vector machines, boosting, etc.) and finding ways to apply them to real-world practical problems in image and signal analysis. He has over 20 publications in the fields of machine learning, genetic algorithms, robotics, evolvable hardware, and computer vision.

John Szymanski received the Ph.D. degree in physics from Carnegie Mellon University, Pittsburgh, PA, in 1987.

He held a Director's Postdoctoral Fellowship at Los Alamos National Laboratory (LANL), Los Alamos, NM, from 1987 to 1990. From 1990 to 1997, he was Assistant Professor of physics at Indiana University, Bloomington. Since 1997, he has been a Technical Staff Member and Project Leader at LANL, where he is Project Leader for the Multispectral Thermal Imager satellite. He has 34 publications in refeered journals, over 30 invited talks presented at conferences, seminars and colloquia, and numerous publications in conference proceedings. 
Jeffrey J. Bloch received the B.S. degree in physics at Massachusetts Institute of Technology, Cambridge, in 1979, and the Ph.D. degree in physics from the University of Wisconsin, Madison, in 1988. His thesis research area involved experimental X-ray astrophysics.

From 1988 to 1990, he was a Postdoctoral Researcher at Los Alamos National Laboratory (LANL), Los Alamos, NM, working on wide area EUV telescopes for astrophysics. In 1991, he became a Staff Member at LANL and in 1993, was Project Leader for the ALEXIS satellite effort. From 1997 to 2000, he was Project Leader for the Deployable Adaptive Processing effort at LANL. From 1998 to 2000, he was Astrophysics and Advanced Applications Team Leader in the Space and Remote Sensing Sciences Group. Since 2000, he has been project leader for the Hyperspectral Infrared Imaging System project at LANL.

Reid B. Porter received the B.S. degree in electronic engineering and information technology and the Ph.D. degree in electronic engineering from the Queensland University of Technology, Australia, in 1996 and 2001, respectively.

$\mathrm{He}$ is currently with Los Alamos National Laboratory, Los Alamos, NM, where he continues to pursue his interests in novel computation architectures for on-board exploitation of large volume data sensors.
Mark Galassi was born in New york, NY, in 1965. He received the B.S. degree in physics from Reed College, Portland, OR, in 1986 and the Ph.D. degree in theoretical physics from State University of New York, Stony Brook, in 1992.

Since then, he has worked at Los Alamos National Laboratory, Los Alamos, NM, as a Staff Research Scientist. His research interests include general relativity, astrophysics (in particular gamma ray bursts), artificial intelligence and evolutionary computation, software engineering, embedded operating systems, and other areas of physics and computer science.

A. Cody Young received the B.S. degree in physics from Colorado College, Colorado Springs, in 1992, and the M.S. degree in physics from the University of Washington (UW), Seattle, in 1999. He is currently pursuing the Ph.D. degree in physics at UW.

Since 1991, he has been with the Los Alamos National Laboratory, Los Alamos, NM, working on various projects, including such diverse topics as dark matter, neutron stars, solar neutrinos, and evolutionary computation. 